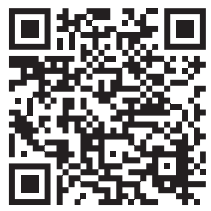
Infección, coronavirus, diabetes.

Keywords: Infection, coronavirus, diabetes.

* Departamento de Instituto Nacional de Cardiología «Ignacio Chávez». Ciudad de México, México.

Recibido: 02/05/2020
Palabras clave: Endocrinología,

\section{Infección por coronavirus en pacientes con diabetes}

\author{
Coronavirus infection in patients with diabetes
}

\author{
Margarita Torres-Tamayo, * Nacú A Caracas-Portillo, ${ }^{*}$ Berenice Peña-Aparicio,* \\ Juan G Juárez-Rojas, ${ }^{*}$ Aida X Medina-Urrutia, ${ }^{*}$ María del R Martínez-Alvarado*
}

\section{RESUMEN}

La diabetes mellitus es una enfermedad crónica, compleja y multifactorial, que se caracteriza por alteración en el metabolismo de la glucosa, las grasas y las proteínas. Con frecuencia, los pacientes que la padecen cursan con hiperglucemia y, además, la enfermedad arterial coronaria es la principal causa de muerte. Las comorbilidades que se asocian con la diabetes son: sobrepeso y obesidad, hipertensión arterial sistémica, dislipidemia aterogénica $\mathrm{y}$, en algunos pacientes, enfermedad vascular periférica, daño renal, neuropatía y retinopatía. El descontrol crónico de la enfermedad se asocia con una mayor susceptibilidad a infecciones, las cuales generalmente cursan con pocos síntomas, aunque, generalmente, la hiperglucemia se magnifica, lo cual empeora el curso de las infecciones. Desde diciembre de 2019, cuando se identificó la enfermedad producida por uno de los coronavirus (coronavirus 2 del síndrome respiratorio agudo grave o SARS-CoV-2), que ha sido llamada enfermedad por coronavirus 2019 (COVID-19) ha habido algunos reportes que asocian la presencia de diabetes con un mayor riesgo de mortalidad. En este artículo de revisión nos hemos enfocado en cuatro puntos específicos: 1) la epidemiología de la prevalencia y de la mortalidad de COVID-19 en la población general y en la población con diabetes mellitus tipo 2;2) la fisiopatología que está relacionada con la unión del SARS-CoV-2 a los receptores en sujetos con diabetes; 3 ) la respuesta inmunológica inducida por el SARS-CoV-2, y 4) el tratamiento ambulatorio y hospitalario que se recomienda en los pacientes con diabetes que se infectan con SARS-CoV-2.

\section{ABSTRACT}

Diabetes mellitus is a complex, multifactorial, chronic disease characterized by impaired metabolism of glucose, fats and proteins. Patients who suffer from it frequently have hyperglycemia and coronary artery disease is the leading cause of death. The comorbidities associated with diabetes are overweight and obesity, systemic arterial hypertension, atherogenic dyslipidemia and in some patients peripheral vascular disease, kidney damage, neuropathy and retinopathy. Chronic lack of control of the disease is associated with increased susceptibility to infections, which generally have few symptoms, but hyperglycemia is generally magnified, which worsens the course of infections. Since December 2019, when the disease caused by one of the coronaviruses (coronavirus 2 of severe acute respiratory syndrome, SARSCoV-2) was identified and has been called coronavirus disease 2019 (COVID-19), there have been some reports that associate the presence of diabetes with an increased risk of mortality. In this review article we have focused on four specific points: 1) epidemiology of the prevalence and mortality of COVID-19 in the general population and in the population with type 2 diabetes mellitus; 2) pathophysiology related to the binding of SARS-CoV-2 to receptors in subjects with diabetes; 3) the immune response induced by SARS-CoV-2, and 4) the outpatient and hospital treatment recommended in patients with diabetes who become infected with SARS-CoV-2. o alta patogenicidad. Los primeros infectan las vías respiratorias superiores y causan un cuadro respiratorio leve o moderado; los segundos (de alta patogenicidad) afectan también las vías respiratorias inferiores, tal como el Middle East respiratory syndrome coronavirus (MERS-CoV) o el severe acute respiratory syndrome coronavirus (SARS-CoV), incluido el tipo 2 (SARS-CoV-2), el cual es causante de la pandemia actual. La
$\delta$-CoV y $\gamma$-CoV ${ }^{1}$ Los CoV que afectan a los humanos se han dividido en aquéllos con baja cual se debe a la presencia de glucoproteínas puntiagudas sobre su envoltura. Existen cuatro géneros diferentes de este virus: $\alpha-\mathrm{CoV}, \beta-\mathrm{CoV}$
I coronavirus (CoV) es un virus-ARN con 
neumonía severa se ha asociado con una rápida replicación viral, infiltración celular inflamatoria masiva y elevación de citocinas y quimiocinas proinflamatorias, las cuales producen daño pulmonar agudo y síndrome de distrés respiratorio agudo (ARDS, del inglés acute respiratory distress syndrome).

Por un lado, en México, la Secretaría de Salud ha reportado que la hipertensión arterial, la diabetes y la obesidad son los tres factores de riesgo más importantemente asociados con la muerte por infección de SARS-CoV-2.* Por otro lado, los sujetos de edad mayor a 65 años con enfermedades preexistentes como hipertensión, diabetes, enfermedad arterial coronaria o cerebrovascular, enfermedad pulmonar obstructiva crónica y enfermedad renal tienen peores desenlaces cuando se infectan con SARS-CoV-2; sin embargo, los mecanismos de esta asociación aún se desconocen.

El presente artículo de revisión aborda los aspectos generales de la infección por SARSCoV-2 y está enfocado hacia los pacientes que presentan diabetes mellitus (DM) tipo 2 .

Los CoV comprenden una gran familia de virus que son comunes en humanos y en animales (camellos, vacas, gatos y murciélagos). En ocasiones, los CoV de animales infectan a los humanos y posteriormente se transmiten de humano a humano, como ha sucedido con el MERS-CoV, el SARS-CoV y, en particular, el SARS-CoV-2, que es causante de la enfermedad conocida como enfermedad por coronavirus 2019 (COVID-19). ${ }^{1}$ En diciembre de 2019, el SARS-CoV-2 se identificó en Wuhan, China, como causante de infecciones del tracto respiratorio inferior. Debido a la rápida diseminación de la COVID-19 en este país y en otras partes del mundo, en marzo de 2020 la Organización Mundial de la Salud (OMS) designó el estado pandémico de la enfermedad. ${ }^{1}$

Aunque el modo de transmisión inicial de SARS-CoV-2 es desconocido, investigaciones epidemiológicas en un mercado de Wuhan sugieren que el inicio de la enfermedad ocurrió por consumo de víboras y, posteriormente, de murciélagos. Conforme la diseminación aumentó, la transmisión de persona a persona y los

\footnotetext{
* (https://coronavirus.gob.mx/datos/, revisado el 20 de abril de 2020).
}

fómites han mostrado ser la principal forma de contagio. ${ }^{2}$ Las secreciones del tracto respiratorio que se expulsan durante los estornudos, la tos o el habla pueden favorecer el contagio entre individuos, debido a que las gotículas de un paciente positivo de SARS-CoV-2 entran en contacto con las mucosas de un sujeto sano. El contacto con una superficie contaminada también puede favorecer el contagio cuando se tocan ojos, nariz o boca, luego de haber tocado dichas superficies. Aunque los pacientes son más contagiosos cuando están sintomáticos, ${ }^{2}$ algunos pueden transmitir la enfermedad aun antes de presentar los síntomas. ${ }^{3,4}$ Se ha propuesto que la otra forma de transmisión de la COVID-19 es el contacto con otros fluidos corporales como la orina y las heces de pacientes infectados, pero esto es menos probable. Aunque también se han descrito dos casos de recién nacidos positivos de SARS-CoV-2 y provenientes de madres también infectadas con el virus, se desconoce el modo de esta transmisión. ${ }^{5,6}$

La tasa de mortalidad del SARS-CoV y MERS-CoV en pacientes embarazadas es de 25 y $40 \%$, respectivamente; con respecto a la mortalidad de COVID-19, sólo se ha reportado un caso de muerte materno/fetal. ${ }^{7}$

\section{ASPECTOS EPIDEMIOLÓGICOS DE LA ENFERMEDAD}

La enfermedad causada por el virus SARSCoV-2 ha mostrado ser una enfermedad que se contagia rápidamente. La severidad de esta enfermedad ha variado desde una gripe autolimitada hasta una neumonía fulminante, que implica falla respiratoria y muerte. La aparición de nuevos casos es mayor en número fuera de China, lo que llevó a la OMS a declarar una pandemia. Según el sitio worldometers.info, el 20 de abril de 2020 había 2,436,811 casos reportados como positivos para COVID-19, con 638,078 pacientes recuperados y 167,278 defunciones en todo el mundo; mientras que en México se reportaron 8,261 casos, con 2,627 pacientes recuperados y 686 muertes.

\section{ESPECTRO DE LA ENFERMEDAD}

La mayoría de las infecciones causadas por SARS-CoV-2 son autolimitadas $(\sim 80 \%)$. La 
enfermedad COVID-19 causa manifestaciones más severas en personas ancianas y en aquéllas con numerosos problemas médicos de fondo. De acuerdo con lo reportado por los CDC (Centers for Disease Control and Prevention) de China, ${ }^{8}$-los cuales incluyen 44,500 infecciones confirmadas - la COVID-19 se presenta de la siguiente manera:

- En forma leve en $81 \%$ de los casos.

- En forma severa en $14 \%$ de los casos (hipoxemia $>50 \%$ de la superficie pulmonar afectada en 24 a 48 horas).

- En forma crítica o severa en 5\% de los casos (falla respiratoria, choque y disfunción multiorgánica).

Su tasa de mortalidad se cifra en $6.8 \%$.

\section{MORBIMORTALIDAD}

Hasta el 5 de marzo de 2020, se ha reportado una tasa de mortalidad de $3.4 \%$ II de un total de 95,333 casos confirmados como positivos para COVID-19 en todo el mundo. ${ }^{9}$ La tasa de mortalidad más baja reportada es de 1.4\%, la cual considera datos de 1,099 pacientes atendidos en 552 hospitales de China continental. ${ }^{10}$

Por otro lado, se investigó la presencia de factores de riesgo de progresión y pronóstico de la enfermedad en una serie de 174 pacientes confirmados con COVID-19. De éstos, los que padecían diabetes y los que no tenían otras comorbilidades $(n=24)$ presentaron un mayor riesgo de neumonía severa. Además, los pacientes con diabetes también se caracterizaron por tener valores más altos de marcadores de inflamación y coagulación; comparados con los sujetos sin diabetes, los pacientes con diabetes tuvieron una prevalencia mayor de enfermedad cardiovascular (32 frente a 14\%), pero una prevalencia menor de fiebre (59.5 frente a $83.2 \%$ ). Los datos bioquímicos mostraron además que los pacientes con diabetes tenían cifras más altas de proteína C reactiva (PCR) (32.8 frente a 16.3), velocidad de sedimentación globular (67 frente a 23) y dímero D (1.15 frente a 0.54). En contraste, la cuenta absoluta de linfocitos (0.86

\footnotetext{
II (Según el reporte de situación núm. 45 de la OMS del 5 de marzo de 2020).
}

frente a 0.97), eritrocitos (3.9 frente a 4.17) y los niveles de hemoglobina (117 frente a 127) fueron significativamente más bajos en el grupo de pacientes con diabetes.

La exclusión de pacientes con otro tipo de comorbilidades mostró que a pesar de no haber diferencia en la sintomatología basal ni en la prevalencia de sexo, los pacientes con diabetes se caracterizaron por ser de mayor edad (61 años frente a 32 años) y tener una frecuencia más alta de náuseas y vómitos. La comparación de las tomografías de tórax expuso que los pacientes con diabetes mostraron alteraciones patológicas más severas que las observadas en los pacientes sin diabetes $(p<0.04)$. Finalmente, el análisis mostró que la mortalidad fue más elevada entre los pacientes con diabetes $(16.3 \%)$ comparados con aquéllos sin diabetes $(0 \%) .{ }^{11}$

\section{INCREMENTO DE LA MORTALIDAD POR COVID-19 ASOCIADA CON DIABETES}

Inicialmente los datos de COVID-19 y diabetes eran escasos y contradictorios. ${ }^{12}$ En un estudio que incluyó 140 pacientes con COVID-19, la diabetes no fue un factor de severidad de la enfermedad. ${ }^{13}$ Asimismo, en otra serie de 150 casos con COVID, Ruan Q. y colaboradores reportaron que no hubo diferencia en la frecuencia de diabetes entre los que fallecieron y los que fueron dados de alta (18 frente a $16 \%, \mathrm{p}=0.88$ ). ${ }^{14}$

No se encontró asociación de los valores de glucosa ni de la presencia de diabetes con la severidad de la enfermedad en el análisis de 11 estudios que evaluaron anormalidades bioquímicas de pacientes con COVID-19. ${ }^{15}$ Por otro lado, en una serie de casos que incluyó 138 pacientes hospitalizados con neumonía por COVID-19, un tercio de ellos tenía factores de riesgo, entre los que estaba la diabetes. ${ }^{16}$ En un reporte de 26 defunciones causadas por COVID-19 en Wuhan, China, se encontró que $42.3 \%$ de las muertes se asoció con la presencia de diabetes. ${ }^{17}$ En contraste, un reporte del CDC chino que incluyó a 72,314 casos con COVID-19 mostró que la mortalidad se incrementaba en sujetos con diabetes, en comparación con aquéllos que no tenían esta enfermedad (7.3 frente a 2.3\%). ${ }^{8}$ Los datos reportados sugieren que los pacientes con diabetes podrían tener mayor riesgo de desarrollar 
complicaciones cuando presentan infección por SARS-CoV-2. No obstante, no parece que la diabetes por sí misma incremente la susceptibilidad de los pacientes a las infecciones. ${ }^{18}$

\section{DIABETES E INFLAMACIÓN}

La diabetes es una enfermedad inflamatoria crónica caracterizada por múltiples alteraciones metabólicas y vasculares que pueden afectar la respuesta a diversos gérmenes. La hiperglucemia y la resistencia a la insulina promueven una mayor síntesis de los productos finales de glucosilación, citocinas proinflamatorias y estrés oxidativo, además estimulan la producción de moléculas de adhesión que median la inflamación. Este proceso inflamatorio puede influir como un mecanismo subyacente que conduce a mayor propensión para infecciones. ${ }^{19}$

La diabetes mal controlada se relaciona con una respuesta inhibida de los linfocitos, así como con un deterioro en el funcionamiento de monocitos, macrófagos y neutrófilos. Además, existe una disfunción tanto en la reacción de hipersensibilidad de tipo retardado como en la activación del complemento en pacientes descompensados. ${ }^{20}$ En modelos animales de diabetes se han observado cambios estructurales en el tejido pulmonar, los cuales están asociados con un aumento de la permeabilidad vascular y con el colapso del epitelio alveolar. ${ }^{21}$ Por su parte, los pacientes con diabetes generalmente presentan una reducción significativa en la capacidad vital forzada y el volumen espiratorio forzado en un segundo. ${ }^{22}$

\section{INFECCIÓN POR CORONAVIRUS}

Tras la exposición, los CoV se unen a las células mediante una proteína «espiga», la cual se escinde por una proteasa de la célula huésped, lo que permite que el virus ingrese y se replique. La enzima convertidora de angiotensina 2 (ECA2), que se expresa ampliamente en el tracto respiratorio, corazón, riñones, intestinos, neuronas cerebrales, endotelio de arterias y venas, células inmunitarias y páncreas ha sido identificada como uno de los principales receptores del SARS-CoV-2. ${ }^{23}$

Los pacientes con COVID-19 comúnmente muestran linfocitopenia $y$, en menor medida, trombocitopenia y leucopenia; estas últimas son las más destacadas entre los pacientes con la forma grave de la enfermedad. Además, se ha reportado que la gravedad de la infección se asocia con los niveles elevados de citocinas proinflamatorias (interleucina [IL] 6, PCR) y con mayor actividad de coagulación, definida por concentraciones elevadas de dímero $\mathrm{D}$ que, adicionalmente, se asocia con un mal pronóstico en los paciente con COVID-19. ${ }^{15}$ En la diabetes, además del marcado proceso inflamatorio, se produce un desequilibrio entre la coagulación y la fibrinólisis, con niveles aumentados de factores de coagulación e inhibición relativa del sistema fibrinolítico (inmunotrombosis). Al mismo tiempo, tanto la resistencia a la insulina como la diabetes están asociadas con la disfunción endotelial y con el aumento en la agregación y activación de las plaquetas, lo que favorece el desarrollo del estado protrombótico y de hipercoagulación. ${ }^{24}$

Estudios en animales infectados con SARSCoV identificaron que la edad avanzada se relaciona con defectos en la función de las células T y células B, con elevados marcadores de inflamación. Esto sugiere que la diabetes sola o en combinación con edad avanzada, hipertensión y/o enfermedades cardiovasculares, las cuales se caracterizan por estados proinflamatorios, pueden contribuir a la replicación del SARS-CoV-2 y a una respuesta proinflamatoria más prolongada, la cual conduciría a una forma de la enfermedad más severa y finalmente más letal. ${ }^{25}$

\section{INFECCIÓN POR CORONAVIRUS Y CONTROL GLUCÉMICO EN PACIENTES CON DIABETES HOSPITALIZADOS}

La infección por SARS-CoV-2 desencadena condiciones de mayor estrés en personas con diabetes, lo que genera un incremento en la liberación de hormonas hiperglucémicas, tales como glucocorticoides y catecolaminas; éstas favorecen el aumento de la variabilidad y la concentración de la glucosa sanguínea. ${ }^{11}$

Un estudio realizado en 29 pacientes con COVID-19 y diabetes mostró que $69 \%$ de los pacientes tuvo valores no deseables de glucosa capilar preprandial y postprandial, además de que $10.3 \%$ sufrió al menos un episodio de hi- 
poglucemia (glucosa $<70 \mathrm{mg} / \mathrm{dL}$ ). ${ }^{26}$ Aunque se ha descrito que la hipoglucemia moviliza monocitos proinflamatorios y aumenta la reactividad plaquetaria (lo cual contribuye a una mayor mortalidad cardiovascular en pacientes con diabetes), se desconoce con precisión el mecanismo de la respuesta inflamatoria e inmunitaria en estos pacientes, así como el efecto de la hiperglucemia y la hipoglucemia sobre la virulencia del SARS-CoV-2 o viceversa. ${ }^{27}$

A pesar de lo antes señalado, Guo y su equipo $^{11}$ reportaron que del total de pacientes con diabetes que usaba insulina antes de su hospitalización por contagio con SARS-CoV-2, cerca de 29.2\% aumentó la dosis de insulina después del egreso y que $37.5 \%$ de los pacientes que tomaban medicamentos orales antes del ingreso comenzó una terapia de insulina después de éste. Lo anterior destaca un control glucémico deficiente en pacientes con diabetes durante la hospitalización por COVID-19. Aunque se ha descrito que los estados inflamatorios agudos y las respuestas agudas al estrés pueden elevar los niveles de glucosa, los autores sugieren que el SARS-CoV-2 puede dañar las células de los islotes pancreáticos. Esta hipótesis estaría apoyada por el estudio realizado durante el primer brote de coronavirus de SARS 2003, en China, en el que se identificó, en 20 de 39 pacientes que no tenían diabetes antes del contagio por SARS, que la diabetes se desarrolló dentro de las dos semanas posteriores a la hospitalización. Aunque el diagnóstico de diabetes pareció ser transitorio, ya que sólo seis pacientes permanecieron con diabetes al egreso y únicamente en dos fue permanente, el estudio post mortem de un paciente mostró una fuerte tinción de la enzima ECA2 en los islotes pancreáticos del paciente con SARS. ${ }^{28}$ De forma anecdótica, Joshua Millers refiere que los pacientes con diabetes e infección por SARS-CoV-2 desarrollan deficiencia insulínica y requieren infusiones de insulina tan altas como 30-40 unidades/hora sin éstas estar relacionadas con el efecto de uso de esteroides. La glucotoxicidad parece estar presente incluso en pacientes con un control glucémico adecuado, lo que se manifiesta desde disglucemia hasta cetoacidosis diabética.

Jean-François Gautier del Hospital Lariboisière (París) reportó que la diabetes de nueva aparición fue descubierta en el contexto de la positividad de la COVID-19, ya que aproximadamente $30 \%$ de los pacientes que ingresaron al hospital con esta enfermedad era portador de diabetes. Aunque la mayoría tenía diabetes tipo 2 , un paciente fue diagnosticado con dificultad respiratoria grave y cetoacidosis diabética con diabetes tipo 1 al requerir terapia con insulina de inmediato. Al parecer, la infección por el SARS-CoV-2 desenmascaró la diabetes.

Aunque actualmente se reconoce que la edad avanzada, la presencia de diabetes, la hipertensión y obesidad severa son promotores de morbilidad y mortalidad en pacientes con COVID-19, ${ }^{29-32}$ se ha reportado que las concentraciones de glucosa en plasma y la presencia de diabetes predicen de manera independiente la morbimortalidad de estos pacientes. ${ }^{14}$ Como se ha descrito previamente, algunos de los mecanismos que podrían contribuir con el aumento en la susceptibilidad de COVID-19 en pacientes con diabetes son los siguientes: a) unión celular de mayor afinidad y entrada de virus eficiente; b) eliminación viral disminuida; c) disminución de la función de las células $T$; d) aumento de la susceptibilidad a la hiperinflamación y el síndrome de tormenta de citocinas y e) presencia de enfermedad cardiovascular. ${ }^{33}$

\section{INMUNIDAD E INFECCIÓN POR SARS-COV-2}

El análisis de las implicaciones de la respuesta inmunitaria en el contexto de la presencia de diabetes mellitus y su respuesta ante una infección viral pueden dar luz respecto al incremento en la severidad de la enfermedad por SARS-CoV-2 en la misma diabetes. La inmunidad innata, que es la primera línea de defensa contra el SARS-CoV-2, se encuentra comprometida durante periodos cortos de hiperglucemia y en pacientes con diabetes descontrolada, lo que permite la proliferación del patógeno en el huésped. ${ }^{20,34}$

Por otro lado, la diabetes se caracteriza por una respuesta exagerada de citocinas proinflamatorias como el factor de necrosis tisular (TNF) y las IL 1 y 6 , lo que puede inducir una respuesta exagerada ante un estímulo, como se ha observado en pacientes con COVID-19 complicados que presentan síndrome de dificultad respiratoria aguda. ${ }^{35}$ Es escasa la 
información que se ha generado durante la pandemia relacionada con este tema, pero en un reporte de 99 casos en Wuhan se encontró un incremento en los neutrófilos totales (38\%), en la IL-6 (52\%) y en la PCR (84\%), mientras que los linfocitos disminuyeron 35\%. ${ }^{36}$ En otro reporte, de 41 pacientes admitidos en un hospital de Wuhan, la mediana de edad fue de 49 años (rango intercuartil: 41-58) y $73 \%$ de los pacientes fue hombres y $32 \%$ tenía comorbilidades (20\% tenía diabetes, 32\% hipertensión y $15 \%$ enfermedad cardiovascular). Todos estos pacientes tuvieron neumonía documentada por tomografía de tórax y la presencia de neutrofilia y linfocitopenia se les asoció con la severidad de la enfermedad y con mortalidad. Además, los pacientes que ingresaron a la unidad de cuidados intensivos ( $\mathrm{UCl}$ ) tuvieron cifras elevadas de citocinas en plasma (IL-2, IL-7, IL-10, factor estimulante de colonias de granulocitos, proteína inducida por interferón [IFN] 10, proteína quimiotáctica de monocitos 1 , proteína inhibidora de monocitos $1 \mathrm{~A}$ y TNF) comparados con aquéllos que no estuvieron en la $\mathrm{UCl}^{36}$

\section{RESPUESTA INMUNITARIA INNATA}

La respuesta inmunitaria innata durante la infección por virus se caracteriza por la expresión del IFN tipo 1 y una cascada de acontecimientos subsecuentes que permiten el control de la replicación viral y la inducción de una respuesta inmunitaria adaptativa efectiva. Durante la infección, el SARS-CoV-2 utiliza como receptor la ECA2, la cual está principalmente expresada en células pulmonares, Ilamadas células alveolares tipo $2 .^{37}$

Algunos coronavirus como el SARS-CoV infectan directamente a macrófagos y células $\mathrm{T}^{35}$ sin embargo, se ignora si el SARS-CoV-2 tiene la capacidad de infectar alguna célula inmunitaria, debido a que solamente un porcentaje mínimo de monocitos/macrófagos expresan ECA2 en el pulmón. No se descarta la existencia de otros receptores que participen durante esta infección. ${ }^{37}$ Para iniciar la respuesta antiviral, las células inmunitarias innatas necesitan reconocer la invasión del virus mediante patrones moleculares frecuentemente asociados con patógenos (PAMP).
Los PAMP contienen ARN genómico viral o ARN intermediario producido durante la replicación y son reconocidos por receptores endosomales de ARN, por el receptor parecido a Toll 3, el receptor parecido a Toll 7 y el sensor citosólico de ARNr, gen inducible de ácido retinoico l/gen asociado con la diferenciación de melanoma 5. Estos eventos de reconocimiento activan la cascada de señalización por medio del factor nuclear- $\mathrm{kB}$ y el factor regulador de IFN 3 con la consecuente translocación nuclear. En el núcleo, estos factores de transcripción inducen la expresión de IFN tipo 1 y otras citocinas proinflamatorias, lo que representa la primera línea de defensa contra la infección viral. El IFN tipo 1 activa la vía JAK-STAT por medio del IFNAR, lo que produce que las cinasas JAK1 y TYK2 fosforilen STAT1 y STAT2. Las STAT1 y 2 se unen con el factor regulador de IFN 9, lo que permite su entrada al núcleo para iniciar la transcripción de genes estimulados por IFN tipo 1 bajo el control del elemento de respuesta estimulado por IFN, el cual contiene promotores; esto finalmente debería suprimir la replicación viral y su diseminación en estadios tempranos de la enfermedad. ${ }^{38}$

La respuesta del IFN tipo 1 a la infección viral por SARS-CoV y MERS-CoV está suprimida. Se ignora si esta supresión también se presenta con el SARS-CoV-2, aunque podría resultar plausible, pero especulativa, debido a la similitud en la secuencia genómica del SARSCoV-2 con el SARS-CoV y el MERS-CoV 79 y $50 \%$, respectivamente). Es posible que en la infección por SARS-CoV-2 ocurra un escenario parecido a la infección letal por SARS-CoV o MERS-CoV, en el cual se ha observado un influjo incrementado de neutrófilos y de monocitosmacrófagos. ${ }^{35,39}$ En un modelo experimental, la causa de neumonía letal fue la desregulación del IFN tipo 1 y de la respuesta inflamatoria de monocitos-macrófagos. ${ }^{40}$

Con base en los datos previos de infección por $\mathrm{CoV}$, la respuesta inmunitaria participa de forma muy importante, por lo que una respuesta tardía o suprimida se asocia con gravedad del daño pulmonar. La replicación viral activa tardía produce un aumento en el IFN tipo I y en el influjo de neutrófilos y macrófagos que desencadenan una «tormenta» de citocinas proinflamatorias. En la infección por SARS- 
CoV-2 la neutrofilia y la linfocitopenia presentes en los pacientes graves posiblemente reflejan un retraso en la respuesta del IFN tipo 1 y en el control viral en la fase temprana de la infección.

\section{RESPUESTA INMUNITARIA ADAPTATIVA}

La respuesta inmunitaria de células Th1 es crucial en la respuesta adaptativa en las infecciones virales. El microambiente de citocinas generadas por células presentadoras de antígenos representa la respuesta de las células $T$. Las células $T$ de ayuda dirigen la respuesta total adaptativa, mientras que las células T citotóxicas son esenciales en la muerte de las células infectadas por el virus. La respuesta inmunitaria humoral —principalmente la producción de anticuerpos neutralizantes - tiene un papel protector al limitar la infección en una fase tardía y previene una futura reinfección. En un estudio, los sueros de cinco pacientes infectados con COVID-19 mostraron reactividad cruzada con el SARS-CoV, pero no con otros CoV. Estos sueros también fueron capaces de neutralizar el SARS-CoV-2 en un ensayo in vitro, lo que sugiere una respuesta humoral adecuada. ${ }^{41}$ En la infección por SARS-CoV se reportó que la respuesta de células T CD8 + fue más frecuente y de mayor magnitud que la de células T CD4 + . Evidencia actual sólida indica que la respuesta tipo Th1 es la clave para el control de las infecciones por SARS-CoV y MERS-CoV, y probablemente también para SARS-CoV-2. La respuesta de células $\mathrm{T}$ CD8+, aunque es crucial, requiere que esté controlada para evitar la patología pulmonar.

\section{MECANISMOS DE EVASIÓN INMUNITARIA}

Algunos reportes indican que los CoV están particularmente adaptados para evadir y disminuir la respuesta inmunitaria humana. Lo anterior podría explicar el largo periodo de incubación de 2-11 días en promedio, comparado con 1-4 días de la influenza. ${ }^{42}$ En resumen, la mayoría de mecanismos inhiben la respuesta inmunitaria innata, especialmente el reconocimiento y la señalización del IFN tipo 1. Las proteínas virales incluyen proteínas de membrana, proteínas no estructurales (NS) y otras proteínas (por ejemplo, NS4a, NS4b y NS15), además de que representan moléculas claves en la modulación de la inmunidad del huésped.

De acuerdo con lo mencionado previamente, el análisis de dos individuos infectados con MERS-CoV con diferente severidad reportó que la respuesta del IFN tipo 1 fue muy inferior en el paciente que murió, comparada con el que se recuperó. ${ }^{43}$ Para la evasión adaptativa inmunitaria, la presentación de antígenos por la vía del complejo mayor de histocompatibilidad clase I y clase II estuvo regulada a la baja cuando macrófagos o células dendríticas se infectaron con MERS-CoV, lo cual podría disminuir marcadamente la activación de células T. ${ }^{44}$

\section{MANEJO Y TRATAMIENTO DE PACIENTES CON DIABETES Y COVID-19}

Informes recientes que fueron realizados en diferentes países sobre la enfermedad COVID-19 indican que la presencia de diabetes está relacionada con una mayor mortalidad y mayor necesidad de cuidados intensivos durante el contagio por dicha enfermedad. ${ }^{45}$ En general, los pacientes con diabetes son más susceptibles a las infecciones, debido a un estado inflamatorio y prooxidativo crónico que impacta negativamente en el perfil glucémico y deteriora la homeostasis glucémica y la sensibilidad periférica a la acción de la insulina. Se desconoce si el descontrol crónico de la diabetes contribuye con la virulencia de la expresión de COVID-19. ${ }^{46}$

Como medida preventiva en este grupo de pacientes, es importante indicar vacunarse contra la influenza y la neumonía. Esto último puede disminuir las posibilidades de adquirir una neumonía bacteriana secundaria después de una infección viral respiratoria, sin embargo, los datos de la epidemia por COVID-19 aún no están disponibles. ${ }^{47}$

Aunque los datos sobre el manejo de la diabetes y la COVID-19 aún son escasos, existen algunas posiciones $y / o$ recomendaciones planteadas por diferentes sociedades médicas. En general, estas recomendaciones pueden aplicarse en países con alto nivel de escolaridad, en donde la mayoría de la población tiene acceso a los servicios de salud y en donde es posible la comunicación vía internet entre los pacientes y los especialistas. 
Los pacientes con diabetes y COVID-19 que presentan síntomas leves a moderados (sin compromiso respiratorio) se pueden atender en el hogar sin la necesidad de realizar visitas físicas al consultorio. Adicionalmente, en las instituciones de salud como el Instituto Mexicano del Seguro Social, la Secretaría de Salud y el Instituto de Seguridad y Servicios Sociales de los trabajadores del Estado se recomienda que los familiares de los pacientes contagiados acudan por las recetas médicas y por los medicamentos. También existe un centro de atención telefónica para que los pacientes que tienen dudas relacionadas con la COVID-19 puedan ser informados y, en caso de ser necesario, instruidos para acudir a las unidades hospitalarias. Finalmente, también existe un porcentaje de pacientes que continúa acudiendo a sus consultas privadas y otros que se ponen en contacto con sus médicos mediante las redes sociales. A continuación, se mencionan algunas recomendaciones que se han establecido en algunos países.

- En países del primer mundo, los pacientes envían mensajes directos a sus médicos tratantes, externando preguntas y preocupaciones. En estos casos, la mayoría de las plataformas de expedientes clínicos ofrecen la opción de registrarse en un portal que se vincula de manera confidencial a la dirección de correo electrónico. También se pueden enviar mediciones de glucosa sanguínea, ya sea trascribiendo o adjuntando las descargas de los medidores de glucosa en sangre capilar o de técnicas de monitoreo continuo de glucosa.

- Se requiere que los sistemas de atención mantengan un mecanismo automatizado o servicio de contestación, ya que muchos pacientes mayores podrían verse desafiados por la tecnología. Un médico de guardia o un profesional de la salud aliado puede ser vital en este respecto.

- Encuentros de telemedicina con interacción audiovisual cara a cara. Tales instancias proporcionan un nivel similar de satisfacción igual al de las visitas reales del paciente y del proveedor, además el examen se presta también a la apariencia general y a la inspección. Estas visitas pueden convertirse en la forma habitual si la pandemia continúa por un periodo prolongado. La Asociación Americana de Diabetes (ADA) publicó recientemente algunas recomendaciones ambulatorias para pacientes con diabetes y COVID-19, las cuales incluyen: ${ }^{48}$

- Beber suficientes líquidos para evitar la deshidratación.

- Mantener niveles de glucosa cercanos a los valores objetivos individualizados.

- Monitorización de los niveles de glucosa en sangre capilar en momentos adicionales al plan cotidiano (durante el día, la noche, al acostarse, etc.) para evitar episodios de hipoglucemia y cetoacidosis.

- Preservar una higiene rigurosa, como lavarse las manos, limpiar las zonas de pinchazos, inyección e infusión mediante agua y jabón o alcohol.

- El tratamiento de las comorbilidades, especialmente de la hipertensión arterial coexistente, la dislipidemia, las enfermedades cardiovasculares y renales no debe interrumpirse.

- Evitar la hospitalización tanto como sea posible. Existen lineamientos para el manejo de pacientes hospitalizados con diabetes, los cuales deben adaptarse para aquellos pacientes que cursan con COVID-19 en las diferentes unidades hospitalarias. ${ }^{27,49}$ Como se mencionó previamente, en un reporte de 29 pacientes hospitalizados con diabetes, el control glucémico no fue adecuado en cerca de $70 \%$ de éstos $(29.4 \%$ de hiperglucemia preprandial y $64.5 \%$ de hiperglucemia posprandial). ${ }^{50}$ Estos resultados sugieren el reto que representa el manejo de los pacientes hospitalizados que padecen diabetes y COVID-19 para que se mantengan óptimos sus niveles de glucosa. Para poder afrontar este desafío se requiere: ${ }^{47-49}$

- Contar con médicos especialistas en los hospitales denominados como COVID para que éstos puedan ser consultados acerca del tratamiento de los fármacos que se utilizan para controlar la glucosa de los pacientes con diabetes.

- Mantener los objetivos de glucemia recomendados por la Asociación Americana de 
Endocrinólogos Clínicos y la ADA (140 mg/ $\mathrm{dL}$ para glucemia preprandial y $180 \mathrm{mg} / \mathrm{dL}$ para glucemia posprandial).

- Manejo de la variabilidad de la glucosa como parte del enfoque integral para controlar la hiperglucemia, principalmente y de forma urgente en $\mathrm{UCl}$.

- Coordinación con los nutriólogos y servicios de comedor de hospitales para ofrecer una dieta integral y equilibrada.

- Proporcionar instrucciones de ejercicio en el interior de las instalaciones.

- Evaluar los niveles de ansiedad de los pacientes (la intervención psicológica debe llevarse a cabo para pacientes necesitados).

- Garantizar las mejores opciones terapéuticas de sostén para el tratamiento de la infección por COVID-19, manteniendo el enfoque en el manejo de pacientes con diabetes y sus posibles complicaciones crónicas, asociadas y agudas.

- Establecer el tratamiento farmacológico con el rigor científico que se requiere mediante los protocolos de investigación.

- Actualmente no se recomienda el uso de monitoreo continuo de la glucosa en el hospital, por lo que este método no se puede recomendar fuera de un ensayo clínico.

El tratamiento farmacológico de los pacientes con diabetes infectados por SARS-CoV-2 será el mismo que en pacientes con diabetes que requieren hospitalización. Las estrategias terapéuticas y los objetivos óptimos de control de la glucosa deben formularse en función de la gravedad de la enfermedad, la presencia de comorbilidades y complicaciones relacionadas con la diabetes, la edad y otros factores. Se debe prestar especial atención a las personas con nefropatía diabética o complicaciones cardiacas relacionadas con la diabetes, ya que corren un mayor riesgo de desarrollar $\mathrm{CO}$ VID-19 grave y de muerte. ${ }^{27}$

Se han reportado algunas consideraciones particulares para el uso en pacientes con diabetes y COVID-19 de ciertos grupos farmacológicos:

- Incretinas. Los análogos del péptido similar al glucagón tipo 1 han mostrado ser beneficiosos en pacientes con diabetes y COVID-19, ya que mejoran el metabolismo de la glucosa y no tienen riesgo de interacciones farmacológicas con el uso de fármacos disponibles contra la infección por SARS-CoV-2; sin embargo, las sociedades médicas en el área sugieren mantener la terapia farmacológica habitual y hacer ajustes sólo en beneficio de la evolución y requerimientos del paciente vigilado intrahospitalariamente. ${ }^{46}$

- Inhibidores de la ECA. Estos fármacos son utilizados con frecuencia en pacientes con diabetes e hipertensión y deberán de continuarse durante la infección por SARS-CoV-2. ${ }^{45,46}$

- Cloroquina e hidroxicloroquina. Es escasa la información relacionada con el uso de estos medicamentos en pacientes con diabetes. Se postula que la cloroquina tiene un amplio potencial antiviral al bloquear la infección y la replicación viral, además de tener un efecto inmunomodulador y antiinflamatorio que ha sido observado en estudios in vitro. ${ }^{45,50-52}$ Aunque los resultados no han sido concluyentes, algunos estudios han mostrado que la hidroxicloroquina mejora el control glucémico en pacientes con diabetes descompensada (promoviendo la reducción de la degradación de insulina intracelular). Si se decide el uso de este tipo de medicamentos, podría ser necesario un ajuste de las dosis de los medicamentos antidiabéticos orales y/o insulina para prevenir posibles eventos hipoglucémicos.

- Corticosteroides. Su efecto en la COVID-19 también está bajo investigación. Como ya se mencionó, el daño pulmonar agudo y el síndrome de distrés respiratorio agudo ocurren, en parte, por la respuesta inmunitaria del huésped. Si bien los corticosteroides suprimen la inflamación pulmonar, también inhiben la inmunidad y la eliminación de patógenos. Actualmente no hay datos disponibles sobre el manejo de pacientes con diabetes infectados por COVID-19. Aunque la hiperglucemia suele ser la principal preocupación en este contexto, no se debe descartar la posibilidad de episodios de hipoglucemia como resultado de la interacción del tratamiento 
farmacológico con la patogénesis viral y con las alteraciones metabólicas típicas de la diabetes. El monitoreo riguroso de la glucosa puede atenuar el empeoramiento de los síntomas y los resultados adversos. La orientación provisional de la OMS sobre el manejo clínico de la infección respiratoria aguda grave cuando se sospecha que hay infección por SARS-CoV-2 limita el uso de corticosteroides fuera de los ensayos clínicos, considerando el efecto hiperglucémico y el impacto de estos fármacos en la respuesta inmunitaria. ${ }^{52-54}$ Por otro lado, la Surviving Sepsis Campaign recomienda el uso de corticosteroides sólo en pacientes graves con ventilación mecánica.

En resumen, la pandemia por COVID-19 es un gran desafío para las personas que viven con diabetes y para el personal de salud asistencial, ya que estos pacientes necesitan atención y cuidados especiales, pues su enfermedad está asociada con una mayor gravedad de los síntomas y las complicaciones. Será necesario un enfoque de equipo multidisciplinario que incluya infectólogos, endocrinólogos, neumólogos, psicólogos, nutriólogos y especialistas en rehabilitación del ejercicio durante los periodos prolongados de hospitalización y recuperación. ${ }^{27}$ Se necesitan más estudios clínicos y fisiopatológicos para establecer más detalles. ${ }^{46}$

Existe un gran temor en la contingencia actual por COVID-19, debido a que no se cuenta con un tratamiento específico contra el virus. El personal de salud se enfrenta a la necesidad de tratar a los pacientes con comorbilidades que los afectan de forma moderada y severa. El Conocimiento generado en otros países, en donde la pandemia inició antes que en México brinda la oportunidad para integrar a los profesionales de la salud (internistas, intensivistas, cardiólogos, nefrólogos, endocrinólogos, infectólogos, enfermeros y nutriólogos), los cuales pueden ofrecer un tratamiento multidisciplinario más completo para los pacientes con diabetes que son afectados por SARS-CoV-2. En estos pacientes, el monitoreo de la glucosa es fundamental, por lo que sería deseable contar con los insumos suficientes para vigilar frecuentemente la glucosa y para permitir una mejor toma de decisiones en el tratamiento de la diabetes.
Hasta el momento, aunque existe plausibilidad en la asociación de diabetes y una mayor frecuencia de infecciones, no hay datos concluyentes de que la prevalencia de diabetes sea mayor en los pacientes infectados. Sin embargo, algunos reportes han documentado una progresión más severa y un riesgo de mortalidad incrementado en 2.3 veces cuando se compara con sujetos sin diabetes. Las medidas que se recomiendan para prevenir la infección por SARS-CoV-2 prácticamente son las mismas que para la población sin diabetes.

\section{REFERENCIAS}

1. Di Gennaro F, Pizzol D, Marotta C, Antunes M, Racalbuto V, Veronese $\mathrm{N}$ et al. Coronavirus diseases (COVID-19) current status and future perspectives: a narrative review. Int J Environ Res Public Health. 2020; 17 (8). doi: 10.3390/ijerph17082690.

2. Joseph T, Ashkan M, editors. COVID-19. International pulmonologist's consensus on COVID-19 [Internet]. International Pulmonologist's Consensus; 2020. Disponible en: file:///C:/Users/Alicia/Downloads/International- PulmonologistsconsensusonCOVID-19.pdf

3. Rothe C, Schunk M, Sothmann P, Bretzel G, Froeschl G, Wallrauch C et al. Transmission of 2019-nCoV infection from an asymptomatic contact in Germany. N Engl J Med. 2020; 382: 970-971.

4. Bai Y, Yao L, Wei T, Tian F, Jin DY, Chen L et al. Presumed asymptomatic carrier transmission of COVID-19. JAMA. 2020 Feb 21. doi: 10.1001/ jama.2020.2565. [Epub ahead of print].

5. BBC News. Coronavirus: Newborn becomes youngest person diagnosed with virus [Internet]. BBC News; 6 de febrero de 2020. Disponible en: https://www. bbc. com/news/world-asia-china-51395655

6. Barone V. Second newborn baby tests positive for coronavirus [Internet]. New York Post; 13 de marzo de 2020. Disponible en: https://nypost.com/2020/03/13/ second-newborn-baby-tests-positive-for-coronavirus

7. Karami P, Nagavi M, Feyzi A, Aghamohammadi M, Novin MS, Mobaien A et al. Mortality of a pregnant patient diagnosed with COVID-19: A case report with clinical, radiological and histopathological findings. Travel Med Infect Dis. 2020; 101665. doi: 10.1016/j. tmaid.2020.101665. [Epub ahead of print].

8. Wu Z, McGoogan JM. Characteristics of and important lessons from the coronavirus disease 2019 (COVID-19) outbreak in China: summary of a report of 72314 cases from the Chinese center for disease control and prevention. JAMA. 2020. doi: 10.1001/jama.2020.2648. [Epub ahead of print].

9. Coronavirus disease 2019 (COVID-19). Situation report - 45. World Health Organization; 5 de marzo de 2020. Disponible en: https://www.who.int/docs/default-source/coronavirus/situation-report/20200305sitrep45

10. Guan WJ, Ni ZY, Hu Y, Liang WH, Ou CQ, He JX et al. Clinical characteristics of coronavirus disease 2019 
in China. N Engl J Med. 2020; 382 (18): 1708-1720.

11. Guo W, Li M, Dong Y, Zhou H, Zhang Z, Tian C et al. Diabetes is a risk factor for the progression and prognosis of COVID-19. Diabetes Metab Res Rev. 2020; e3319. doi: 10.1002/dmrr.3319. [Epub ahead of print].

12. Gupta R, Ghosh A, Singh AK, Misra A. Clinical considerations for patients with diabetes in times of COVID-19 epidemic. Diabetes Metab Syndr. 2020; 14 (3): 211-212.

13. Zhang JJ, Dong $X$, Cao YY, Yuan YD, Yang YB, Yan YQ et al. Clinical characteristics of 140 patients infected with SARS-CoV-2 in Wuhan, China. Allergy. 2020 Feb 19. doi: 10.1111/all.14238. [Epub ahead of print].

14. Ruan Q, Yang K, Wang W, Jiang L, Song J. Clinical predictors of mortality due to COVID-19 based on an analysis of data of 150 patients from Wuhan, China. Intensive Care Med. 2020 Apr 6. doi: 10.1007/ s00134020-06028-z. [Epub ahead of print].

15. Lippi G, Plebani M. Laboratory abnormalities in patients with COVID-2019 infection. Clin Chem Lab Med. 2020. doi: 10.1515/cclm-2020-0198. [Epub ahead of print].

16. Wang D, Hu B, Hu C, Zhu F, Liu X, Zhang J et al. Clinical characteristics of 138 hospitalized patients with 2019 novel coronavirus-infected pneumonia in Wuhan, China. JAMA. 2020; 323: 1061.

17. Deng SQ, Peng HJ. Characteristics of and public health responses to the coronavirus disease 2019 outbreak in China. J Clin Med. 2020; 9 (2): 575.

18. Knapp S. Diabetes and infection: is there a link? - A mini-review. Gerontology. 2013; 59 (2): 99-104.

19. Abregú AV, Carrizo TR, Díaz El, Velarde MS, Fonio MC, Bazán MC. Subclinical inflammation in children and adolescents with type 1 diabetes. Acta Bioquim Clin Latinoam. 2015; 49: 393-398.

20. Geerlings SE, Hoepelman AIM. Immune dysfunction in patients with diabetes mellitus (DM). FEMS Immunol Med Microbiol. 1999; 26: 259-265.

21. Popov D, Simionescu M. Alterations of lung structure in experimental diabetes, and diabetes associated with hyperlipidaemia in hamsters. Eur Respir J. 1997; 10 (8): 1850-188

22. Lange P, Groth S, Kastrup J, Mortensen J, Appleyard M, Nyboe J et al. Diabetes mellitus, plasma glucose and lung function in a cross-sectional population study. Eur Respir J. 1989; 2 (1): 14-19.

23. Wu C, Chen X, Cai Y, Xia J, Zhou X, Xu S et al. Risk factors associated with acute respiratory distress syndrome and death in patients with coronavirus disease 2019 pneumonia in Wuhan, China. JAMA Intern Med. 2020. doi: 10.1001/jamainternmed.2020.0994. [Epub ahead of print].

24. Bikdeli B, Madhavan MV, Jimenez D, Chuich T, Dreyfus I, Driggin E et al. COVID-19 and thrombotic or thromboembolic disease: implications for prevention, antithrombotic therapy, and follow-up. J Am Coll Cardiol. 2020. doi: 10.1016/j.jacc.2020.04.031. [Epub ahead of print].

25. Li B, Yang J, Zhao F, Zhi L, Wang X, Liu L et al. Prevalence and impact of cardiovascular metabolic diseases on COVID-19 in China. Clin Res Cardiol. 2020; 109 (5): 531-538.
26. Zhou J, Tan J. Diabetes patients with COVID-19 need better blood glucose management in Wuhan, China. Metabolism. 2020; 107: 154216.

27. Hussain A, Bhowmik B, do Vale Moreira NC. COVID-19 and diabetes: Knowledge in progress. Diabetes Res Clin Pract. 2020; 162: 108142.

28. Yang JK, Lin SS, Ji XJ, Guo LM. Binding of SARS coronavirus to its receptor damages islets and causes acute diabetes. Acta Diabetol. 2010; 47 (3): 193-199.

29. Onder G, Rezza G, Brusaferro S. Case-fatality rate and characteristics of patients dying 273 in relation to COVID-19 in Italy. JAMA. 2020. doi: 10.1001/ jama.2020.4683. [Epub ahead of print].

30. Centers for Disease Control and Prevention. National Diabetes Statistics Report, 2020 [Internet]. Atlanta, GA: US Department of Health and Human Services; 2020. Disponible en: https://www.cdc.gov/diabetes/ library/features/diabetes-stat-report.html

31. Yang J, Zheng Y, Gou X, Pu K, Chen Z, Guo Q et al. Prevalence of comorbidities in the novel Wuhan coronavirus (COVID-19) infection: a systematic review and meta-analysis. Int J Infect Dis. 2020; 94: 91-95.

32. Yang X, Yu Y, Xu J, Shu H, Xia J, Liu H et al. Clinical course and outcomes of critically ill patients with SARSCoV-2 pneumonia in Wuhan, China: a single-centered, retrospective, observational study. Lancet Respir Med. 2020 Feb 24. doi: 10.1016/S2213-2600(20)30079-5. [Epub ahead of print].

33. Muniyappa R, Gubbi S. COVID-19 pandemic, coronaviruses, and diabetes mellitus. Am J Physiol Endocrinol Metab. 2020; 318 (5): E736-E741.

34. Fraser C, Riley S, Anderson RM, Ferguson NM. Factors that make an infectious disease outbreak controllable. Proc Natl Acad Sci USA. 2004; 101: 6146-6151.

35. Perlman S, Dandekar AA. Immunopathogenesis of coronavirus infections: implications for SARS. Nat Rev Immunol. 2005; 5 (12): 917-927.

36. Huang $C$, Wang $Y$, Li X, Ren L, Zhao J, Hu Y et al. Clinical features of patients infected with 2019 novel coronavirus in Wuhan, China. Lancet. 2020; 395 (10223): 497-506.

37. Zhu N, Zhang D, Wang W, Li X, Yang B, Song J et al. A novel coronavirus from patients with pneumonia in China, 2019. N Engl J Med. 2020; 382: 727-733.

38. de Wit E, van Doremalen N, Falzarano D, Munster VJ. SARS and MERS: recent insights into emerging coronaviruses. Nat Rev Microbiol. 2016; 14: 523-534.

39. Zumla A, Hui DS, Perlman S. Middle east respiratory syndrome. Lancet. 2015; 386: 995-1007.

40. Channappanavar R, Perlman S. Pathogenic human coronavirus infections: causes and consequences of cytokine storm and immunopathology. Semin Immunopathol. 2017; 39: 529-539.

41. Zhou P, Yang XL, Wang XG, Hu B, Zhang L, Zhang W et al. A pneumonia outbreak associated with a new coronavirus of probable bat origin. Nature. 2020; 579 (7798): 270-273.

42. Lessler J, Reich NG, Brookmeyer R, Perl TM, Nelson KE, Cummings DA. Incubation periods of acute respiratory viral infections: a systematic review. Lancet Infect Dis. 2009; 9: 291-300.

43. Faure E, Poissy J, Goffard A, Fournier C, Kipnis E, Titecat $\mathrm{M}$ et al. Distinct immune response in two MERS-CoV- 
infected patients: can we go from bench to bedside? PLoS One. 2014; 9: e88716.

44. Shokri S, Mahmoudvand S, Taherkhani R, Farshadpour F. Modulation of the immune response by Middle East respiratory syndrome coronavirus. J Cell Physiol. 2019; 234: 2143-2151.

45. Sanders JM, Monoque ML, Jodlowski TZ, Cutrell JB. Pharmacologic treatments for coronavirus disease 2019 (COVID-19): A Review. JAMA. 2020. doi: 10.1001/jama.2020.6019. [Epub ahead of print].

46. Stolan AP, Banerjee Y, Rizvi AA, Rizzo M. Diabetes and the COVID-19 pandemic: How insights from recent experience might guide future management. Metab Syndr Relat Disord. 2020; 18 (4): 173-175.

47. Gupta R, Ghosh A, Singh AK, Misra A. Clinical considerations for patients with diabetes in times of COVID-19 epidemic. Diabetes Metab Syndr. 2020; 14 (3): 211-212.

48. Moghissi ES, Korytkowski MT, DiNardo M, Einhorn D, Hellman R, Hirsch IB et al. American Association of Clinical Endocrinologists and American Diabetes Association consensus statement on inpatient glycemic control. Diabetes Care. 2009; 32 (6): 1119-1131.

49. Miazgowski T, Bikowska M, Ogonowski J, Taszarek A. The impact of health locus of control and anxiety on self-monitored blood glucose con- centration in women with gestational diabetes mellitus. J Womens Health (Larchmt). 2018; 27 (2): 209-215.

50. Gautret P, Lagier JC, Parola P, Hoang VT, Meddeb L, Mailhe $M$ et al. Hydroxychloroquine and azithromycin as a treatment of COVID-19: results of an open-label non-randomized clinical trial. Int J Antimicrob Agents. 2020; 105949. doi: 10.1016/j. ijantimicag.2020.105949. [Epub ahead of print].

51. Singh AK, Sing HA, Shaikh A, Singh R, Misra A. Chloroquine and hydroxychloroquine in the treatment of COVID-19 with or without diabetes: A systematic search and a narrative review with a special reference to India and other developing countries. Diabetes Metab Syndr. 2020; 14 (3): 241-246.

52. Klonoff D, Umpierrez G. COVID-19 in patients with diabetes: risk factors that increase morbidity.
Metabolism. 2020; 154224. doi: 10.1016/j. metabol.2020.154224. [Epub ahead of print].

53. Zhao ZW, Zhang FC, Xu M, Huang K, Zhong WN, Cai WP et al. Clinical analysis of 190 cases of outbreak with atypical pneumonia in Guangzhou in spring, 2003. Zhonghua Yixue Zazhi. 2003; 83 (9): 713-718.

54. Meng QH, Dong PL, Guo YB, Zhang K, Liang LC, Hou $\mathrm{W}$ et al. Use of glucocorticoid in treatment of severe acute respiratory syndrome cases. Zhonghua Yufang Yixue Zazhi. 2003; 37 (4): 233-235.

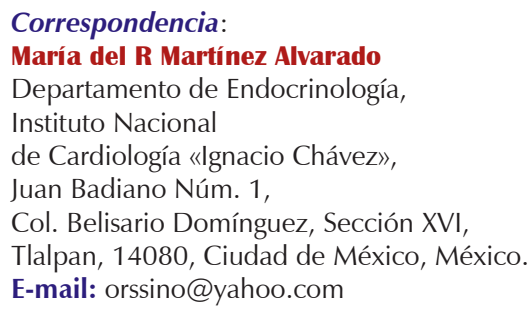

\section{RESPONSABILIDADES ÉTICAS}

Protección de personas y animales. Los autores declaran que para esta investigación no se han realizado experimentos en seres humanos ni en animales.

Confidencialidad de los datos. Los autores declaran que han seguido los protocolos de su centro de trabajo sobre la publicación de datos de pacientes.

Derecho a la privacidad y consentimiento informado. Los autores han obtenido el consentimiento informado de los pacientes $y / o$ sujetos referidos en el artículo. Este documento obra en poder del autor de correspondencia. 